Review Article

\title{
Two Versions of the Projection Postulate: From EPR Argument to One-Way Quantum Computing and Teleportation
}

\author{
Andrei Khrennikov \\ School of Mathematics and Systems Engineering, International Center of Mathematical Modeling in \\ Physics and Cognitive Sciences, Växjö University, 35195, Sweden \\ Correspondence should be addressed to Andrei Khrennikov, andrei.khrennikov@vxu.se
}

Received 17 August 2009; Accepted 29 December 2009

Academic Editor: Shao-Ming Fei

Copyright (c) 2010 Andrei Khrennikov. This is an open access article distributed under the Creative Commons Attribution License, which permits unrestricted use, distribution, and reproduction in any medium, provided the original work is properly cited.

\begin{abstract}
Nowadays it is practically forgotten that for observables with degenerate spectra the original von Neumann projection postulate differs crucially from the version of the projection postulate which was later formalized by Lüders. The latter (and not that due to von Neumann) plays the crucial role in the basic constructions of quantum information theory. We start this paper with the presentation of the notions related to the projection postulate. Then we remind that the argument of Einstein-Podolsky-Rosen against completeness of QM was based on the version of the projection postulate which is nowadays called Lüders postulate. Then we recall that all basic measurements on composite systems are represented by observables with degenerate spectra. This implies that the difference in the formulation of the projection postulate (due to von Neumann and Lüders) should be taken into account seriously in the analysis of the basic constructions of quantum information theory. This paper is a review devoted to such an analysis.
\end{abstract}

\section{Introduction}

We recall that for observables with nondegenerate spectra the two versions of the projection postulate, see von Neumann [1] and Lüders [2], coincide. We restrict our considerations to observables with purely discrete spectra. In this case each pure state is projected as the result of measurement onto another pure state, the corresponding eigenvector. Lüders postulated that the situation is not changed even in the case of degenerate spectra; see [2]. By projecting a pure state we obtain again a pure state, the orthogonal projection on the corresponding eigen-subspace. However, von Neumann pointed out that in general the postmeasurement state is not pure, it is a mixed state. The difference is crucial! And it is surprising that so little attention was paid up to now to this important problem. It is especially surprising if one takes into account the fundamental role which is played by the projection postulate in quantum 
information (QI) theory. QI is approaching the stage of technological verification and the absence of a detailed analysis of the mentioned problem is a weak point in its foundations.

This paper is devoted to such an analysis. We start with a short recollection of the basic facts on the projection postulates and conditional probabilities in QM. Then we analyze the EPR argument against completeness of QM [3]. Since Einstein et al. proceeded on the physical level of rigorousness, it is a difficult task to extract from their considerations which version of the projection postulate was used. We did this in $[4,5]$. Now we shortly recall our previous analysis of the EPR argument. We will see that they really applied the Lüders postulate. They used the fact that a measurement on a composite system transforms a pure state into another pure state, the orthogonal projection of the original state. The formal application of the original von Neumann postulate blocks the EPR considerations completely.

We analyze the quantum teleportation scheme. We will see again that it is fundamentally based on the use of the Lüders postulate. The formal application of the von Neumann postulate blocks the teleportation type schemes; see for more detail [6].

Finally, we remark that "one way quantum computing," for example, [7-9] (an exciting alternative to the conventional scheme of quantum computing) is irreducibly based on the use of the Lüders postulate.

The results of this analysis ought to be an alarm signal for people working in the quantum foundations. If one assumes that von Neumann was right, but Lüders as well as Einstein et al. were wrong, then all basic schemes of QI should be reanalysed. However, a deeper study of von Neumann's considerations on the projection postulate [1] shows that, in fact, under quite natural conditions the von Neumann postulate implies the Lüders postulate. The detailed (rather long and technical) proof of this unexpected result can be found in preprint [10]. In this paper we just formulate the above mentioned conditions and the theorem on the reduction of one postulate to another. Thus the basic QI schemes seem to be save in their appealing to the Lüders version of the projection postulate. However, following additional analysis is still needed to understand the adequacy of conditions of a theorem on the reduction of one postulate to another to the original considerations of von Neumann in his book [1]. He wrote on the physical level of rigorousness. To make a mathematically rigorous reformulation of his arguments is not an easy task!

The main conclusion of the present paper is that the study of the foundations of QM and QI is far from being completed; see also the recent monograph of Jaeger [11]. (We can also point to the recent study on teleportation of Asano et al. [12]. It is the teleportation scheme in the infinite-dimensional Hilbert space, known as Kossakowski-Ohya scheme. It would be interesting to analyze this scheme to understand the role of the projection postulate in its realization. We emphasize that measurements on composite systems play the crucial point of QI.) We remark that the operational approach to QM, see, for example, [13], considers not only the von Neumann and Lüders versions of the projection postulate, but general theory of postmeasurement states. Formally, one may say that from the viewpoint of the operational approach it is not surprising that, for example, the von Neumann postulate can be violated for some measurement. It is neither surprising that even both projection postulates can be violated. But this viewpoint is correct only on the level of formal mathematical considerations. If we turn to the real physical situation, that is, experiments, we should carefully analyze concrete experiments to understand which type of postmeasurement state is produced. Finally, we mention the viewpoint of De Muynck $[14,15]$ who emphasized that all projection type postulates are merely about conditional probabilities. In principle, I agree with him, compare with my recent monograph [16]. However, experimenters are interested 
not only in probabilities of results of measurements, but also in the postmeasurement states. We can mention the quantum teleportation schemes or one-way quantum computing.

\section{Projection Postulate}

\subsection{Nondegenerate Case}

Everywhere below $H$ denotes a complex Hilbert space. Let $\psi \in H$ be a pure state, that is, $\|\psi\|^{2}=1$. We remark that any pure state induces a density operator

$$
\widehat{\rho}_{\psi}=\psi \otimes \psi=\widehat{P}_{\psi}
$$

where $\widehat{P}_{\psi}$ denotes the orthogonal projector on the vector $\psi$. This operator describes an ensemble of identically prepared systems each of them in the same state $\psi$.

For an observable $A$ represented by the operator $\widehat{A}$ with nondegenerate spectrum von Neumann's and Lüders' projection postulates coincide. For simplicity we restrict our considerations to operators with purely discrete spectra. In this case the spectrum consists of eigenvalues $\alpha_{k}$ of $\widehat{A}: \widehat{A} e_{k}=\alpha_{k} e_{k}$. Nondegeneracy of the spectrum means that subspaces consisting of eigenvectors corresponding to different eigenvalues are one dimensional. The following definition was formulated by von Neumann [1] in the case of nondegenerate spectrum. It coincides with Lüders' definition (we remain once again that Lüders' did not distinguish the cases of degenerate and nondegenerate spectra).

PP: Let $A$ be an observable described by the self-adjoint operator $\widehat{A}$ having purely discrete nondegenerate spectrum. Measurement of observable $A$ on a system in the (pure) quantum state $\psi$ producing the result $A=\alpha_{k}$ induces transition from the state $\psi$ into the corresponding eigenvector $e_{k}$ of the operator $\widehat{A}$.

If we select only systems with the fixed measurement result $A=\alpha_{k}$, then we obtain an ensemble described by the density operator $\widehat{q}_{k}=e_{k} \otimes e_{k}$. Any system in this ensemble is in the same state $e_{k}$. If we do not perform selections, we obtain an ensemble described by the density operator

$$
\widehat{q}_{\psi}=\sum_{k}\left|\left\langle\psi, e_{k}\right\rangle\right|^{2} \widehat{P}_{e_{k}}=\sum_{k}\left\langle\widehat{\rho}_{\psi} e_{k}, e_{k}\right\rangle \widehat{P}_{e_{k}}=\sum_{k} \widehat{P}_{e_{k}} \widehat{\rho}_{\psi} \widehat{P}_{e_{k}}
$$

where $\widehat{P}_{e_{k}}$ is the projector on the eigenvector $e_{k}$.

\subsection{Degenerate Case}

Lüders generalized this postulate to the case of operators having degenerate spectra. Let us consider the spectral decomposition for a self-adjoint operator $\widehat{A}$ having purely discrete spectrum

$$
\widehat{A}=\sum_{i} \alpha_{i} \widehat{P}_{i}
$$


where $\alpha_{i} \in \mathbf{R}$ are different eigenvalues of $\widehat{A}$ (so $\alpha_{i} \neq \alpha_{j}$ ) and $\widehat{P}_{i}, i=1,2, \ldots$, is the projector onto subspace $H_{i}$ of eigenvectors corresponding to $\alpha_{i}$.

By Lüders' postulate after a measurement of an observable $A$ represented by the operator $\widehat{A}$ that gives the result $\alpha_{i}$ the initial pure state $\psi$ is transformed again into a pure state, namely,

$$
\psi_{i}=\frac{\widehat{P}_{i} \psi}{\left\|\widehat{P}_{i} \psi\right\|} .
$$

Thus for the corresponding density operator we have

$$
\widehat{Q}_{i}=\psi_{i} \otimes \psi_{i}=\frac{\widehat{P}_{i} \psi \otimes \widehat{P}_{i} \psi}{\left\|\widehat{P}_{i} \psi\right\|^{2}}=\frac{\widehat{P}_{i} \widehat{P}_{\psi} \widehat{P}_{i}}{\left\|\widehat{P}_{i} \psi\right\|^{2}} .
$$

If one does not make selections corresponding to the values $\alpha_{i}$ the final postmeasurement state is given by

$$
\widehat{q}_{\psi}=\sum_{i} p_{i} \widehat{Q}_{i}, \quad p_{i}=\left\|\widehat{P}_{i} \psi\right\|^{2},
$$

or simply

$$
\widehat{q}_{\psi}=\sum_{i} \widehat{q}_{i}, \quad \widehat{q}_{i}=\widehat{P}_{i} \rho_{\psi} \widehat{P}_{i}
$$

This is the statistical mixture of the pure states $\psi_{i}$. Thus by Lüders there is no essential difference between measurements of observables with degenerate and nondegenerate spectra.

von Neumann had a completely different viewpoint on the postmeasurement state [1]. Even for a pure state $\psi$ the postmeasurement state (for a measurement with selection with respect to a fixed result $A=\alpha_{k}$ ) will not be a pure state again. If $\widehat{A}$ has degenerate (discrete) spectrum, then according to von Neumann [1].

A measurement of an observable A giving the value $A=\alpha_{i}$ does not induce a projection of $\psi$ on the subspace $H_{i}$.

The result will not be a fixed pure state, in particular, not Lüders' state $\psi_{i}$. Moreover, the postmeasurement state, say $\widehat{g}_{\psi}$, is not uniquely determined by the formalism of QM! Only a subsequent measurement of an observable $D$ such that $A=f(D)$ and $\widehat{D}$ is an operator with nondegenerate spectrum (refinement measurement) will determine the final state.

Following von Neumann, we choose an orthonormal basis $\left\{e_{\text {in }}\right\}$ in each $H_{i}$. Let us take a sequence of real numbers $\left\{\gamma_{\text {in }}\right\}$ such that all numbers are distinct. We define the corresponding self-adjoint operator $\widehat{D}$ having eigenvectors $\left\{e_{\text {in }}\right\}$ and eigenvalues $\left\{\gamma_{\text {in }}\right\}$ :

$$
\widehat{D}=\sum_{i} \sum_{n} \gamma_{\mathrm{in}} \widehat{P}_{e_{\mathrm{in}}}
$$


A measurement of the observable $D$ represented by the operator $\widehat{D}$ can be considered as a measurement of the observable $A$, because $A=f(D)$, where $f$ is some function such that $f\left(\gamma_{\text {in }}\right)=\alpha_{i}$. The $D$-measurement (without postmeasurement selection with respect to eigenvalues) produces the statistical mixture

$$
\widehat{O}_{D ; \psi}=\sum_{i} \sum_{n}\left|\left\langle\psi, e_{\text {in }}\right\rangle\right|^{2} \widehat{P}_{e_{\text {in }}}
$$

By selection for the value $\alpha_{i}$ of $A$ (its measurements realized via measurements of a refinement observable $D$ ) we obtain the statistical mixture described by normalization of the operator

$$
\widehat{O}_{i, D ; \psi}=\sum_{n}\left|\left\langle\psi, e_{\text {in }}\right\rangle\right|^{2} \widehat{P}_{e_{\text {in }}}
$$

von Neumann emphasized that the mathematical formalism of QM could not describe in a unique way the postmeasurement state for measurements (without refinement) in the case of degenerate observables. He did not discuss the properties of such states directly, he described them only indirectly via refinement measurements. (For him this state was a kind of hidden variable. It might even be that he had in mind that it "does not exist at all," i.e., it could not be described by a density operator.) We would like to proceed by considering this (hidden) state under the assumptions that it can be described by a density operator, say $\hat{g}_{\psi}$. We formalize a list of properties of this hidden (postmeasurement) state each of which can be extracted from von Neumann's considerations on refinement measurements. Finally, we prove, see Theorem 5.3, that $\widehat{g}_{\psi}$ should coincide with the postmeasurement state postulated by Lüders in [2].

Consider the $A$-measurement without refinement. By von Neumann, for each quantum system $s$ in the initial pure state $\psi$, the $A$-measurement with the $\alpha_{i}$-selection transforms the $\psi$ in one of states $\phi=\phi(s)$ belonging to the eigensubspace $H_{i}$. Unlike Lüders' approach, it implies that, instead of one fixed state, namely, $\psi_{i} \in H_{i}$, such an experiment produces a probability distribution of states on the unit sphere of the subspace $H_{i}$.

\section{3. von Neumann's Viewpoint on the EPR Experiment}

Consider any composite system $s=\left(s_{1}, s_{2}\right)$. Consider any $H_{1}=H_{2}=L_{2}\left(R^{3}, d x\right)$. Let $a_{1}$ and $a_{2}$ be observables represented by the operators $\widehat{a}_{1}$ and $\widehat{a}_{2}$ with purely discrete nondegenerate spectra:

$$
\widehat{a}_{i} e_{i}^{\alpha}=\lambda_{i}^{\alpha} e_{i}^{\alpha}, \quad i=1,2
$$

Any state $\psi \in H=H_{1} \otimes H_{2}$ can be represented as

$$
\psi=\sum_{\alpha, \beta} c_{\alpha \beta} e_{1}^{\alpha} \otimes e_{2}^{\beta}
$$


where $\sum_{\alpha, \beta}\left|c_{\alpha \beta}\right|^{2}=1$. Einstein, Podolsky, and Rosen claimed that measurement of $A_{1}$ given by

$$
\widehat{A}_{1}=\widehat{a}_{1} \otimes I
$$

induces a projection of $\psi$ onto one of states $e_{1}^{\alpha} \otimes u, u \in H_{2}$.

In particular, for a state of the form

$$
\psi=\sum_{\gamma} c_{\gamma} e_{1}^{\gamma} \otimes e_{2}^{\gamma}
$$

one of states $e_{1}^{\gamma} \otimes e_{2}^{\gamma}$ is created.

Thus by performing a measurement on the $s_{1}$ with the result $\lambda_{1}^{\gamma}$ the "element of reality"

$$
a_{2}=\lambda_{2}^{\gamma}
$$

is assigned to $s_{2}$. This is the crucial point of the considerations of Einstein et al. [3]. Now by selecting another observable, say $b_{2}$ acting on $s_{2}$, we can repeat our considerations for the operators $\widehat{a}_{1} \otimes \widehat{b}_{2}$. This operator induces another decomposition of the state $\psi$. Another element of reality can be assigned to the same system $s_{2}$. If the operators $\widehat{a}_{2}$ and $\widehat{b}_{2}$ do not commute, then the observables $a_{2}$ and $b_{2}$ are incompatible. Nevertheless, EPR was able to assign to the system $s_{2}$ elements of reality corresponding to these obervables. This contradicts to the postulate of QM that such an assignment is impossible (because of Heisenberg uncertainty relations). To resolve this paradox EPR proposed that QM is incomplete, that is, in spite of Heisenberg's uncertainty relation, two elements of reality corresponding to incompatible observables can be assigned to a single system. As an absurd alternative to incompleteness, they considered the possibility of action at distance. By performing a measurement on $s_{1}$ we change the state of $s_{2}$ and assign it a new element of reality.

However, the EPR considerations did not match von Neumann's projection postulate, because the spectrum of $\widehat{A}_{1}$ is degenerate. Thus by von Neumann to obtain an element of reality one should perform a measurement of a "nonlocal observable" $A$ given by a nonlocal refinement of, for example, $\widehat{A}_{1}=\widehat{a}_{1} \otimes I$ and $\widehat{A}_{2}=I \otimes \widehat{a}_{2}$.

Finally, (after considering of operators with discrete spectra) Einstein et al. considered operators of position and momentum having continuous spectra. According to the von Neumann [1] one should proceed by approximating operators with continuous spectra by operators with discrete spectra.

In Section 5 we will show that under quite natural conditions von Neumann postulate implies Lüders postulate, even for observables with degenerate spectrum. It will close "loophole" in the EPR considerations.

\section{4. von Neumann's Viewpoint on the Canonical Teleportation Scheme}

We will proceed across the quantum teleportation scheme, see, for example, [11], and point to applications of the projection postulate. In this section following the QI-tradition we will use Dirac's symbols to denote the states of systems. There are Alice $(A)$ and Bob $(B)$, and 
Alice has a qubit in some arbitrary quantum state $|\psi\rangle$. Assume that this quantum state is not known to Alice and she would like to send this state to Bob. Suppose Alice has a qubit that she wants to teleport to Bob. This qubit can be written generally as $|\psi\rangle=\alpha|0\rangle+\beta|1\rangle$.

The quantum teleportation scheme requires Alice and Bob to share a maximally entangled state before, for instance, one of the four Bell states: $\left|\Phi^{+}\right\rangle=(1 / \sqrt{2})\left(|0\rangle_{A} \otimes|0\rangle_{B}+\right.$ $\left.|1\rangle_{A} \otimes|1\rangle_{B}\right),\left|\Phi^{-}\right\rangle=(1 / \sqrt{2})\left(|0\rangle_{A} \otimes|0\rangle_{B}-|1\rangle_{A} \otimes|1\rangle_{B}\right),\left|\Psi^{+}\right\rangle=(1 / \sqrt{2})\left(|0\rangle_{A} \otimes|1\rangle_{B}+|1\rangle_{A} \otimes|0\rangle_{B}\right)$, $\left|\Psi^{-}\right\rangle=(1 / \sqrt{2})\left(|0\rangle_{A} \otimes|1\rangle_{B}-|1\rangle_{A} \otimes|0\rangle_{B}\right)$. Alice takes one of the particles in the pair, and Bob keeps the other one. We will assume that Alice and Bob share the entangled state $\left|\Phi^{+}\right\rangle$. So, Alice has two particles (the one she wants to teleport, and $A$, one of the entangled pair), and Bob has one particle, $B$. In the total system, the state of these three particles is given by

$$
|\psi\rangle \otimes\left|\Phi^{+}\right\rangle=(\alpha|0\rangle+\beta|1\rangle) \otimes \frac{1}{\sqrt{2}}(|0\rangle \otimes|0\rangle+|1\rangle \otimes|1\rangle) .
$$

Alice will then make a partial measurement in the Bell basis on the two qubits in her possession. To make the result of her measurement clear, we will rewrite the two qubits of Alice in the Bell basis via the following general identities (these can be easily verified): $|0\rangle \otimes|0\rangle=(1 / \sqrt{2})\left(\left|\Phi^{+}\right\rangle+\left|\Phi^{-}\right\rangle\right),|0\rangle \otimes|1\rangle=(1 / \sqrt{2})\left(\left|\Psi^{+}\right\rangle+\left|\Psi^{-}\right\rangle\right),|1\rangle \otimes|0\rangle=(1 / \sqrt{2})\left(\left|\Psi^{+}\right\rangle-\left|\Psi^{-}\right\rangle\right)$, $|1\rangle \otimes|1\rangle=(1 / \sqrt{2})\left(\left|\Phi^{+}\right\rangle-\left|\Phi^{-}\right\rangle\right)$. Evidently the result of her (local) measurement are that the three-particle state would collapse to one of the following four states (with equal probability of obtaining each): $\left|\Phi^{+}\right\rangle \otimes(\alpha|0\rangle+\beta|1\rangle),\left|\Phi^{-}\right\rangle \otimes(\alpha|0\rangle-\beta|1\rangle),\left|\Psi^{+}\right\rangle \otimes(\beta|0\rangle+\alpha|1\rangle)$, $\left|\Psi^{-}\right\rangle \otimes(-\beta|0\rangle+\alpha|1\rangle)$. The four possible states for Bob's qubit are unitary images of the state to be teleported. The crucial step, the local measurement done by Alice on the Bell basis, is done. It is clear how to proceed further. Alice now has complete knowledge of the state of the three particles; the result of her Bell measurement tells her which of the four states the system is in. She simply has to send her results to Bob through a classical channel. Two classical bits can communicate which of the four results she obtained. After Bob receives the message from Alice, he will know which of the four states his particle is in. Using this information, he performs a unitary operation on his particle to transform it to the desired state $\alpha|0\rangle+\beta|1\rangle$.

If Alice indicates that her result is $\left|\Phi^{+}\right\rangle$, Bob knows that his qubit is already in the desired state and does nothing. This amounts to the trivial unitary operation, the identity operator.

If the message indicates $\left|\Phi^{-}\right\rangle$, Bob would send his qubit through the unitary gate given by the Pauli matrix $\sigma_{3}=\left[\begin{array}{cc}1 & 0 \\ 0 & -1\end{array}\right]$ to recover the state. If Alice's message corresponds to $\left|\Psi^{+}\right\rangle$, Bob applies the gate $\sigma_{1}=\left[\begin{array}{ll}0 & 1 \\ 1 & 0\end{array}\right]$ to his qubit. Finally, for the remaining case, the appropriate gate is given by $\sigma_{3} \sigma_{1}=i \sigma_{2}=\left[\begin{array}{cc}0 & 1 \\ -1 & 0\end{array}\right]$. Teleportation is therefore achieved.

The main problem is that Alice's measurement is represented by a degenerate operator in the 3-qubit space. It is nondegenerate with respect to her 2 quibits, but not in the total space. Thus the standard conclusion that by obtaining, for example, $A=1$, Alice can be sure that Bob obtained the right state $|\psi\rangle$, does not match the quantum measurement theory. According to von Neumann, to get this state Bob should perform a refinement measurement. In order to perform it, Bob should know the state $|\psi\rangle$. Thus from von Neumann's viewpoint there is a loophole in the quantum teleportation scheme. It will be closed (under quite natural conditions) in the next section. 


\section{Reduction of von Neumann's Postulate to Lüders' Postulate}

In this section we try to formalize von Neumann's considerations on the measurement of observables with degenerate spectra.

Consider an $A$-measurement without refinement. By von Neumann, for each quantum system $s$ in the initial pure state $\psi$, the $A$-measurement with the $\alpha_{i}$-selection transforms $\psi$ in one of the states $\phi=\phi(s)$ belonging to the eigensubspace $H_{i}$. This implies that, instead of one fixed state, namely, $\psi_{i} \in H_{i}$, such an experiment produces a probability distribution of states on the unit sphere of the subspace $H_{i}$.

We postulate (it is one of the steps in the formalization of von Neumann's considerations).

DO: For any value $\alpha_{i}$ such that $\widehat{P}_{i} \psi \neq 0$, the postmeasurement probability distribution on $H_{i}$ can be described by a density operator, say $\widehat{\Gamma}_{i}$.

Here $\widehat{\Gamma}_{i}: H_{i} \rightarrow H_{i}$ is such that $\widehat{\Gamma}_{i} \geq 0$ and $\operatorname{Tr} \widehat{\Gamma}_{i}=1$. Consider now the corresponding density operator $\widehat{G}_{i}$ in $H$. Its restriction on $H_{i}$ coincides with $\widehat{\Gamma}_{i}$. In particular this implies its property

$$
\widehat{G}_{i}\left(H_{i}\right) \subset H_{i} .
$$

We remark that $\widehat{G}_{i}$ is determined by $\psi$, so $\widehat{G}_{i} \equiv \widehat{G}_{i \psi \psi}$.

We would like to present the list of other properties of $\widehat{G}_{i}$ induced by von Neumann's considerations on refinement. Since, for each refinement measurement $D$, the operators $\widehat{A}$ and $\widehat{D}$ commute, the measurement of $A$ with refinement can be performed in two ways. First we perform the $D$-measurement and then we get $A$ as $A=f(D)$. However, we also can first perform the $A$-measurement, obtain the postmeasurement state described by the density operator $\widehat{G}_{i}$, then measure $D$ and, finally, we again find $A=f(D)$.

Take an arbitrary $\phi \in H_{i}$ and consider a refinement measurement $D$ such that $\phi$ is an eigenvector of $\widehat{D}$. Thus $\hat{D} \phi=\gamma_{\phi} \phi$. Then for the cases-[direct measurement of $D$ ] and [first $A$ and then $D$ ] —we get probabilities which are coupled in a simple way. In the first case (by Born's rule)

$$
\mathbf{P}\left(D=\gamma_{\phi} \mid \hat{\rho}_{\psi}\right)=|\langle\psi, \phi\rangle|^{2}
$$

In the second case, after the $A$-measurement, we obtain the state $\widehat{G}_{i}$ with probability

$$
\mathbf{P}\left(A=\alpha_{i} \mid \hat{\rho}_{\psi}\right)=\left\|\widehat{P}_{i} \psi\right\|^{2}
$$

Performing the $D$-measurement for the state $\widehat{G}_{i}$ we get the value $\gamma_{\phi}$ with probability

$$
\mathbf{P}\left(D=\gamma_{\phi} \mid \widehat{G}_{i}\right)=\operatorname{Tr} \widehat{G}_{i} \widehat{P}_{\phi}
$$


By (classical) Bayes' rule, we have

$$
\mathbf{P}\left(D=\gamma_{\phi} \mid \hat{\rho}_{\psi}\right)=\mathbf{P}\left(A=\alpha_{i} \mid \hat{\rho}_{\psi}\right) \mathbf{P}\left(D=\gamma_{\phi} \mid \widehat{G}_{i}\right) .
$$

Finally, we obtain

$$
\mathbf{P}\left(D=\gamma_{\phi} \mid \widehat{G}_{i}\right)=\operatorname{Tr} \widehat{G}_{i} \widehat{P}_{\phi}=\frac{|\langle\psi, \phi\rangle|^{2}}{\left\|\widehat{P}_{i} \psi\right\|^{2}} .
$$

Thus

$$
\operatorname{Tr} \widehat{G}_{i} \widehat{P}_{\phi}=\frac{|\langle\psi, \phi\rangle|^{2}}{\left\|\widehat{P}_{i} \psi\right\|^{2}}
$$

This is one of the basic features of the postmeasurement state $\widehat{G}_{i}$ (for the $A$-measurement with the $\alpha_{i}$-selection, but without any refinement). Another basic equality we obtain in the following way. Take an arbitrary $\phi^{\prime} \in H_{i}^{\perp}$, and consider a measurement of the observable described by the orthogonal projector $\widehat{P}_{\phi^{\prime}}$ under the state $\widehat{G}_{i}$. Since the later describes a probability distribution concentrated on $H_{i}$, we have

$$
\mathbf{P}\left(P_{\phi^{\prime}}=1 \mid \widehat{G}_{i}\right)=0
$$

Thus

$$
\operatorname{Tr} ; \widehat{G}_{i} \widehat{P}_{\phi^{\prime}}=0 \text {. }
$$

This is the second basic feature of the postmeasurement state. Our aim is to show that (5.7) and (5.9) imply that, in fact,

$$
\widehat{G}_{i}=\frac{\widehat{P}_{i} \widehat{\rho}_{\psi} \widehat{P}_{i}}{\left\|\widehat{P}_{i} \psi\right\|^{2}} \equiv \frac{\widehat{P}_{i} \psi \otimes \widehat{P}_{i} \psi}{\left\|\widehat{P}_{i} \psi\right\|^{2}},
$$

that is, to derive Lüders postulate which is a theorem in our approach.

Lemma 5.1. The postmeasurement density operator $\widehat{G}_{i}$ maps $H$ into $H_{i}$.

Proof. By (5.1) it is sufficient to show that $\widehat{G}_{i}\left(H_{i}^{\perp}\right) \subset H_{i}$. By (5.9) we obtain

$$
\left\langle\widehat{G}_{i} \phi^{\prime}, \phi^{\prime}\right\rangle=0
$$


for any $\phi^{\prime} \in H_{i}^{\perp}$. This immediately implies that $\left\langle\widehat{G}_{i} \phi_{1}^{\prime}, \phi_{2}^{\prime}\right\rangle=0$ for any pair of vectors from $H_{i}^{\perp}$. The latter implies that $\widehat{G}_{i} \phi^{\prime} \in H_{i}$ for any $\phi^{\prime} \in H_{i}^{\perp}$.

Consider now the $A$-measurement without refinement and selection. The postmeasurement state $\widehat{g}_{\psi}$ can be represented as

$$
\widehat{g}_{\psi}=\sum_{m} p_{m} \widehat{G}_{m}, \quad p_{m}=\left\|\widehat{P}_{m} \psi\right\|^{2}
$$

Proposition 5.2. For any pure state $\psi$ and self-adjoint operator $\widehat{A}$ with purely discrete (degenerate) spectrum the postmeasurement state (in the absence of a refinement measurement) can be represented as

$$
\widehat{g}_{\psi}=\sum_{m} \widehat{g}_{m}
$$

where $\widehat{g}_{m}: H \rightarrow H_{m}, \widehat{g}_{m} \geq 0$, and, for any $\phi \in H_{m}$,

$$
\left\langle\widehat{g}_{m} \phi, \phi\right\rangle=|\langle\psi, \phi\rangle|^{2}
$$

Theorem 5.3. Let $\widehat{g} \equiv \widehat{g}_{\psi}$ be a density operator described by Proposition 5.2. Then

$$
\widehat{g}_{m}=\widehat{P}_{m} \psi \otimes \widehat{P}_{m} \psi
$$

\section{Conclusion}

We performed a comparative analysis of two versions of the projection postulate-due to von Neumann and Lüders. We recalled that for observables with degenerate spectra these versions imply consequences which at least formally different. In the case of a composite system any measurement on a single subsystem is represented by an operator with degenerate spectrum. Such measurements play the fundamental role in quantum foundations and quantum information: from the original EPR argument to shemes of quantum teleportation and quantum computing. We formulated natural conditions reducing the von Neumann projection postulate to the Lüders projection postulate; see the theorem. This theorem closed mentioned loopholes in QI-schemes. However, conditions of this theorem are the subject of further analysis.

\section{References}

[1] J. von Neumann, Mathematische Grundlagen der Quantenmechanik, Springer, Berlin, Germany, 1932.

[2] G. Lüders, “Uber die Zustansanderung durch den Messprozess," Ann. Phys. Lpz., vol. 8, p. 322, 1951.

[3] A. Einstein, B. Podolsky, and N. Rosen, "Can quantum-mechanical description of physical reality be considered complete?" Physical Review, vol. 47, p. 777, 1935.

[4] A. Khrennikov, "The role of von Neumann and Lüders postulates in the Einstein, Podolsky, and Rosen considerations: comparing measurements with degenerate and nondegenerate spectra," Journal of Mathematical Physics, vol. 49, no. 5, Article ID 052102, 5 pages, 2008. 
[5] A. Khrennikov, "EPR "Paradox", projection postulate, time synchronization "nonlocality"," International Journal of Quantum Information, vol. 7, no. 1, pp. 71-78, 2009.

[6] A. Khrennikov, "Analysis of the role of von Neumann's projection postulate in the canonical scheme of quantum teleportation," Journal of Russian Laser Research, vol. 29, no. 3, pp. 296-301, 2008.

[7] R. Raussendorf and H. J. Briegel, "A one-way quantum computer," Physical Review Letters, vol. 86, no. 22, pp. 5188-5191, 2001.

[8] G. Vallone, E. Pomarico, F. De Martini, and P. Mataloni, "One-way quantum computation with twophoton multiqubit cluster states," Physical Review A, vol. 78, no. 4, Article ID 042335, 2008.

[9] N. C. Menicucci, S. T. Flammia, and O. Pfister, "One-way quantum computing in the optical frequency comb," Physical Review Letters, vol. 101, no. 13, Article ID 130501, 2008.

[10] A. Khrennikov, "The role of von Neumann and Lüders postulates in the EPR-Bohm-Bell considerations: did EPR make a mistake?" http:/ /arxiv.org/abs/0801.0419.

[11] G. Jaeger, Quantum Information: An Overview, Springer, New York, NY, USA, 2007.

[12] M. Asano, M. Ohya, and Y. Tanaka, "Complete m-level teleportation based on Kossakowski-Ohya scheme," in Proceedings of QBIC-2, Quantum Probability and White Noise Analysis, vol. 24, pp. 19-29, 2009.

[13] M. Ozawa, "Conditional probability and a posteriori states in quantum mechanics," Publications of Research Institute for Mathematical Sciences, vol. 21, no. 2, pp. 279-295, 1985.

[14] W. M. De Muynck, "Interpretations of quantum mechanics, and interpretations of violations of Bell's inequality," Quantum Probability and White Noise Analysis, vol. 13, pp. 95-104, 2001.

[15] W. M. De Muynck, Foundations of Quantum Mechanics, an Empiricists Approach, Kluwer Academic Publishers, Dodrecht, The Netherlands, 2002.

[16] A. Khrennikov, Contextual Approach to Quantum Formalism, Springer, Berlin, Germany, 2009. 


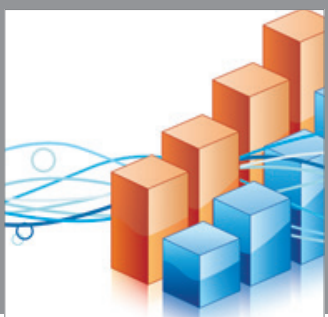

Advances in

Operations Research

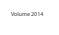

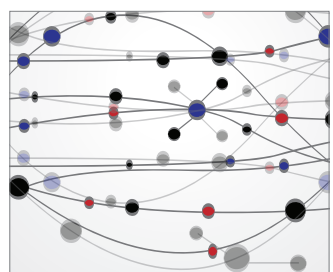

\section{The Scientific} World Journal
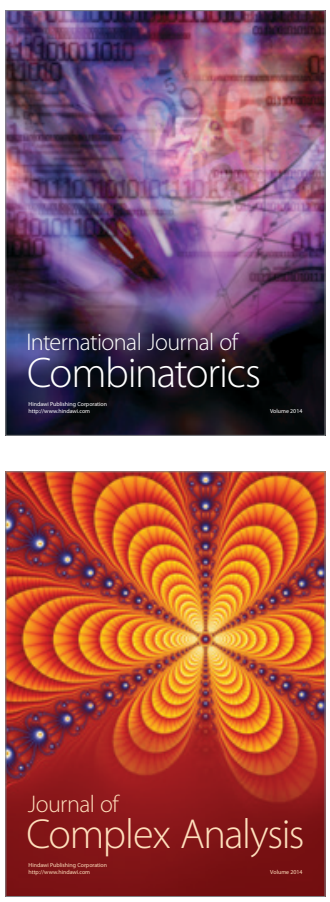

International Journal of

Mathematics and

Mathematical

Sciences
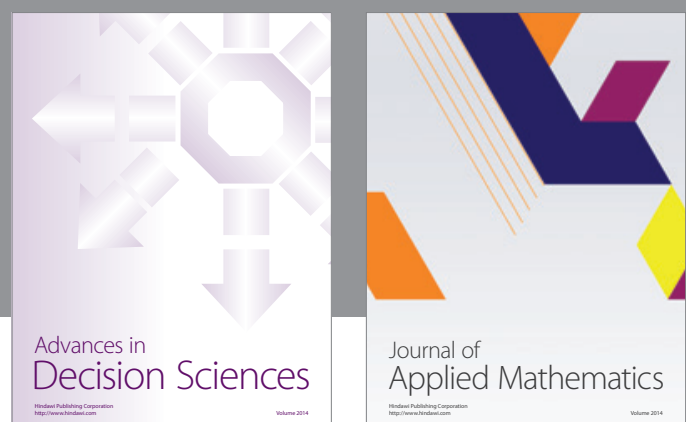

Journal of

Applied Mathematics
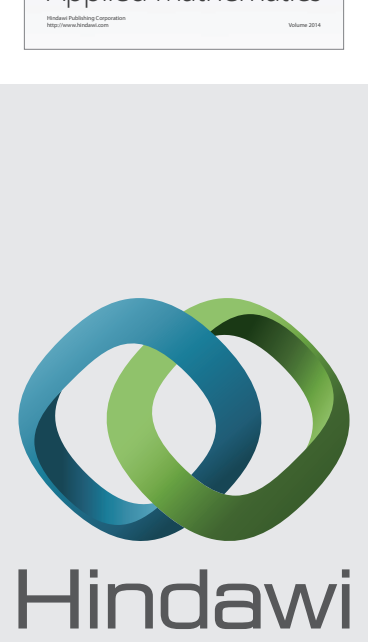

Submit your manuscripts at http://www.hindawi.com
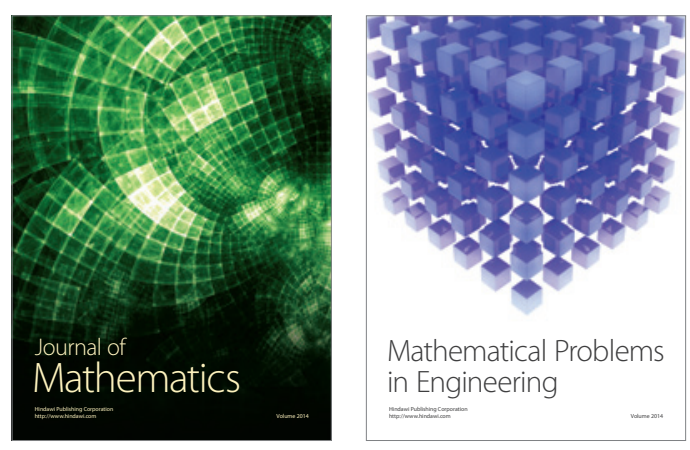

Mathematical Problems in Engineering
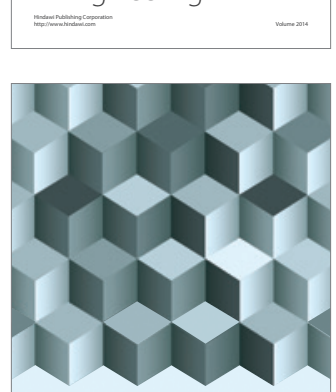

Journal of

Function Spaces
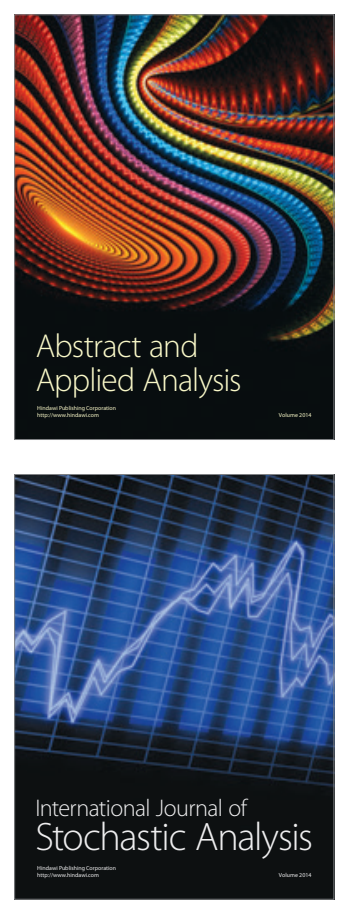

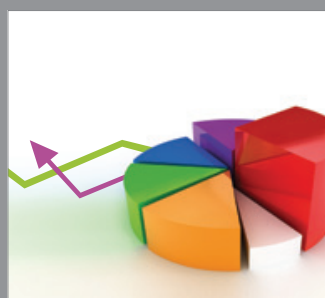

ournal of

Probability and Statistics

Promensencen
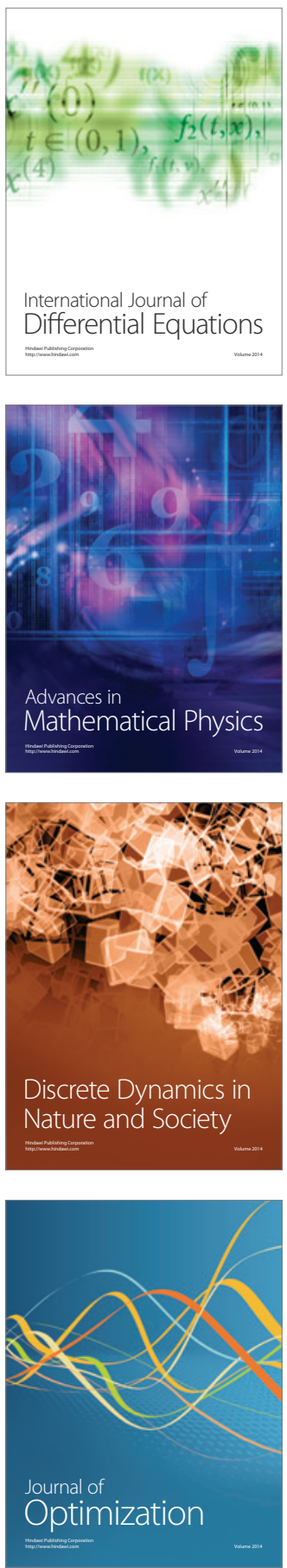